\title{
Dosimétrie du personnel en milieu hospitalier*
}

\author{
B. AUBERT**, D. PAUL***, R. SABATTIER****
}

(Reçu le 11 mars 1997, accepté le 14 mars 1997),

RÉSUMÉ Le personnel du milieu médical représente la majorité $(70 \%)$ des travailleurs exposés aux rayonnements lonisants. Leur exposition est très variable selon les pratiques et les domaines concernés. Nous nous sommes intéressés dans ce travail à l'exposition externe en abordant successivement les concepts du passage de la dose à l'équivalent de dose, les principaux dosimètres (actifs et passifs) disponibles, l'origine de l'exposition et, enfin, l'exposition individuelle au travers de la dosimétrie réglementaire et de quelques études de dosimétrie opérationnelle. Actuellement les informations issues de la dosimétrie réglementaire sont rassurantes puisque $93 \%$ des travailleurs sont exposés à des niveaux inférieurs au niveau d'enregistrement des films dosimètres $(0,2 \mathrm{mSv})$ et $98 \%$ à moins de $5 \mathrm{mSv} / \mathrm{an}$. Cependant des études en médecine nucléaire et surtout en radiologie interventionnelle montrent la nécessité d'un rappel constant des bonnes pratiques de radioprotection, le besoin de relais entre la personne compétente et les services concernés et leur intérêt pour la mise en auvre d'une démarche d'optimisation.

ABSTRACT Workers in the medical field make up the majority (70 \%) of those working under ionising radiation. Their exposure is due to pratices and applications in the field. In this paper we focussed on external exposure by tackling successively the concepts from dose to dose equivalent, the main dosimeters (active and passive) available, the exposure sources and, then, the individugl exposure estimated from the legal dosimetry and from some studies in operational dosimetry. At this moment data from legal dosimetry are reassuring because $93 \%$ of workers are exposed to levels lower than the registration level of the film badge $(0.2$ mSv) and $98 \%$ to less than $5 \mathrm{mSv} /$ year. Nevertheless, some studies in Nuctear Medicine and especially in Interventional Radiology show the need for constant information on the good practices in radiation protection, the necessity of a link between the radiation protection adviser and the concerned departments and the interest to undertake an optimisation process.

\footnotetext{
* Cet article est le dernier d'une série initialement prévue en publication dans un numéro spécial consacré à la dosimétrie.

** Service de physique, Institut Gustave-Roussy, F-94805 Villejuif Cedex.

*** Institut de protection et de sûreté nucléaire, Département de protection de la santé de l'Homme et de dosimétrie, Service de dosimétrie, B.P. 6, F-92265 Fontenay-aux-Roses Cedex.

**** Service de Radiothérapie, Centre Hospitalier Régional, B.P. 6709, F-45067 Orléans Cedex 02.
} 


\section{Introduction}

L'exposition professionnelle en milieu hospitalier dépend bien sûr directement du type de sources de rayonnements ionisants utilisées et de la façon de les appliquer qu'il s'agisse d'un but thérapeutique ou diagnostique. Ces sources, qui sont très diverses, émettent d'une façon générale des photons $\mathrm{X}$ et gamma, des électrons de haute énergie, des particules bêta ou encore des neutrons et sont utilisés dans les principaux domaines suivants : radiothérapie externe, curiethérapie, radiologie, médecine nucléaire et laboratoires de radioanalyse. Toutes ces applications entraînent pour le personnel un risque d'exposition externe, ainsi que pour les deux derniers domaines un risque d'exposition interne en raison de l'utilisation de sources non scellées. Nous ne rapporterons ici que des informations concernant la dosimétrie externe car c'est elle qui concerne le plus grand nombre de travailleurs et pour laquelle existent le plus de données.

Le personnel du milieu médical représente, en France, la majorité de la population des travailleurs exposés aux rayonnements ionisants $(70 \%$ sur plus de 187000 travailleurs affectés à des travaux sous rayonnement en 1995, hors ceux appartenant à des exploitants nucléaires autorisés à effectuer leur propre dosimétrie : CEA, EDF, ...). Ce personnel est réparti dans plus de 15000 établissements ou cabinets. Les données les plus complètes proviennent de la dosimétrie réglementaire, basée sur l'exposition du film dosimètre porté à la poitrine. Pour certaines activités, et souvent les plus irradiantes, ces résultats sont loin de refléter la réalité, surtout pour l'exposition des extrémités. Il est donc nécessaire de pratiquer une dosimétrie opérationnelle complémentaire, adaptée aux différentes utilisations des sources en milieu médical.

Nous allons aborder successivement des considérations générales visant à rappeler des notions fondamentales concernant le passage de la mesure à l'équivalent de dose, les grandeurs utilisées en radioprotection, les sources à l'origine de l'exposition externe, les principaux dosimètres actifs et passifs rencontrés en milieu hospitalier à des fins de dosimétrie réglementaire et/ou opérationnelle et enfin les données de la surveillance individuelle à l'échelle nationale et au travers d'études de cas pour les situations les plus irradiantes.

\section{Passage de la mesure à l'équivalent de dose}

En milieu hospitalier par rapport au domaine de l'industrie nucléaire, le problème n'est pas tant celui de la détection des rayonnements, en général bien caractérisés à l'exception de quelques cas particuliers liés aux traitements à haute énergie (les photoneutrons produits par les accélérateurs linéaires par exemple), mais plutôt celui de la dosimétrie du personnel. En effet, si la détection a beaucoup été explorée, la dosimétrie, en particulier la dosimétrie individuelle des travailleurs du milieu médical, fait intervenir un grand nombre de paramètres compliquant le passage de la mesure à la dose et présente beau- 
coup d'équations à plusieurs inconnues qui ne conduisent qu'à une estimation de la dose.

\subsection{Grandeurs opérationnelles en radioprotection}

De quelle dose parle-t-on en radioprotection? En ce qui concerne la dosimétrie individuelle, l'ICRU(1) dans ses recommandations (ICRU, 1985, 1988, 1992, 1993) a introduit le concept de « grandeurs opérationnelles » avec parmi elles l'équivalent de dose individuel : $H_{\mathrm{p}}(d)$, représentation pratique de l'équivalent de dose efficace $H_{\mathrm{E}}$ ou de l'équivalent de dose peau $H_{\text {peau }}$. Ces dernières grandeurs dosimétriques dites « primaires ", et définies par l'ICRP(2) (1977) sont non mesurables. Depuis 1985, l'ICRU propose la mise en place d'une dosimétrie « pratique " avec des grandeurs modélisées et mesurables dites opérationnelles $\left(H_{\mathrm{p}}(d)\right.$ pour la dosimétrie individuelle mais aussi $H^{\prime}(d, \Omega)$ et $H^{*}(d)$ pour la dosimétrie d'ambiance). Celles-ci permettent d'estimer les grandeurs de l'ICRP 26 (1977) (sans sous-estimation notable) avec l'instrumentation existante pour tous les rayonnements dans un domaine où les doses sont très inférieures aux limites admissibles définies également par l'ICRP(3) (1977).

Par définition (ICRU 47), $H_{\mathrm{p}}(d)$ est l'équivalent de dose dans le tissu mou sous un point spécifié du corps, à une profondeur appropriée $(d)$ dans le champ de rayonnement réel. Pour les rayonnements fortement pénétrants, la profondeur recommandée est $10 \mathrm{~mm} ; H_{\mathrm{p}}(10)$ « représente » alors $H_{\mathrm{E}}$. Pour les rayonnements faiblement pénétrants, la profondeur recommandée est $0,07 \mathrm{~mm}$; $H_{\mathrm{p}}(0,07)$ représente alors $H_{\text {peau. }}$

\subsection{Aspects pratiques du passage à la dose}

Les dosimètres individuels, qu'ils soient actifs ou passifs sont dits " relatifs" pour les distinguer des dosimètres absolus utilisés par les métrologistes. Ces derniers présentent une relation directe (rapport proportionnel) entre une grandeur mesurable (quantité d'énergie apparaissant sous forme thermique, nombre de molécules ou de radicaux nouveaux pour une réaction radio-chimique, charge libérée par l'ionisation d'un gaz) et la dose absorbée dans le tissu prise comme référence légale. Les dosimètres relatifs sont étalonnés sur fantôme par rapport à cette référence qui peut être aussi le kerma dans l'air ou

\footnotetext{
(1) ICRU : International commission on radiation units and measurements.

(2) ICRP : International commission on radiological protection.

(3) En 1991, l'ICRP dans sa publication $n^{\circ} 60$ (1991) a défini de nouvelles grandeurs primaires: $E$, la dose efficace (ou effective) et $H_{\mathrm{T}}$, la dose équivalente à l'organe $\mathrm{T}$, selon un nouveau concept de doses moyennes à l'organe multipliées par des facteurs de pondération $W_{\mathrm{R}}$. Ces grandeurs étant non mesurables puisque non définies en un point contrairement à l'ICRP 26 (1977), leur représentation par les grandeurs. opérationnelles de l'TCRU 47 mesurées en un point est raisonnablement correcte si le facteur de qualité $Q(L)$ est utilisé au lieu de $W_{\mathrm{R}}\left(\right.$ ICRP 60 , p. 88). Pour les photons et les électrons, $Q(L) \approx W_{\mathrm{R}}=1$, ce qui implique que $H_{\mathrm{E}}$ est peu différent de $E$ pour ces types de rayonnement.
} 
dans les tissus, ou encore la fluence. Un facteur de conversion, calculé, est ensuite appliqué pour convertir la grandeur de référence en $H_{\mathrm{p}}(10)$. Ce facteur dépend du spectre au point de mesure.

La mesure des grandeurs opérationnelles serait simple si les spectres aux postes de travail autour des installations médicales étaient connus et si les dosimètres couramment employés avaient des réponses angulaires et énergétiques conformes à la définition de $H_{\mathrm{p}}(10)$ donnée par l'ICRU. En pratique, les dosimètres opérationnels comme les thermoluminescents et les films présentent des caractéristiques acceptables pour déterminer $H_{\mathrm{p}}(10)$. En revanche, les conditions ne sont pas remplies pour les dosimètres cylindriques (type stylodosimètre) dont la réponse n'est pas « isodirectionnelle » mais isotrope.

\section{L'IEC(1) recommande pour les photons que :}

- le rapport entre une lecture à un angle $\alpha$ et une lecture à $\alpha=0^{\circ}$ pour des angles allant de $+75^{\circ}$ à $-75^{\circ}$ varie au plus de $\pm 20 \%$ à l'énergie du ${ }^{137} \mathrm{Cs}$ $(662 \mathrm{keV})$ et de $\pm 50 \%$ à l'énergie du ${ }^{241} \mathrm{Am}(60 \mathrm{keV})$;

- la réponse en énergie en terme de $H_{\mathrm{p}}(10)$ varie au plus de $\pm 30 \%$ pour des photons d'énergie comprise entre $50 \mathrm{keV}$ et $1,5 \mathrm{MeV}$ (référence : ${ }^{137} \mathrm{Cs}$ );

- la mesure de $H_{\mathrm{p}}(10)$ varie au plus de $\pm 50 \%$, avec des particules bêta d'énergie maximale comprise entre 2 et $3,5 \mathrm{MeV}$.

Plusieurs dosimètres permettent de mesurer l'équivalent de dose dans les tissus adjacents au badge à $0,07,3$ (œil) et $10 \mathrm{~mm}$ de profondeur. Les dosimètres thermoluminescents qui sont de bons "équivalents-tissus " pour les photons ont des réponses angulaires et en énergie proches de l'idéal grâce à l'adjonction de filtres. Leur volume sensible est cependant encore trop épais pour la dosimétrie bêta. Les films photographiques avec filtres sont toujours utilisés bien qu'étant de mauvais équivalents-tissus. L'indépendance énergétique pour différents angles d'incidence est en général difficile à assurer surtout aux basses énergies pour les photons (radiothérapie superficielle, radiodiagnostic, ...).

L'étalonnage des dosimètres se fait en présence d'un fantôme adéquat qui reproduit le rayonnement diffusé par le corps de l'individu, contribution intégrée dans la définition de $H_{\mathrm{p}}(10)$. La méthode fait l'objet d'une norme ISO(2). L'ICRU 47 recommande le fantôme PMMA (polyméthylmétacrylate) de $30 \mathrm{~cm}$ $\times 30 \mathrm{~cm} \times 15 \mathrm{~cm}$ dont la masse est proche de la sphère ICRU et dont les caractéristiques de rétrodiffusion sont proches de celles du corps humain. L'ISO préconise un fantôme de $30 \mathrm{~cm} \times 30 \mathrm{~cm} \times 15 \mathrm{~cm}$ rempli d'eau avec des parois en PMMA (frontale : 2,5 mm d'épaisseur, autres : $10 \mathrm{~mm}$ ).

(1) IEC : International electro technical commission.

(2) ISO : International standards organization. 
En France, l'étalonnage des films dosimètres par l'OPRI(1) s'effectue au moyen de 2 faisceaux : cobalt 60 et rayons $X$ de $80 \mathrm{kV}$ (filtration $4 \mathrm{~mm} \mathrm{Be}+$ $1 \mathrm{~mm} \mathrm{Al}$ ). Les conditions d'étalonnage sont proches de celles correspondant à la mesure de $H_{\mathrm{p}}(10)$.

\subsection{Dosimètres individuels en milieu hospitalier}

Les rayonnements ionisants auxquels le personnel peut être exposé en milieu hospitalier sont essentiellement des photons gamma ou des rayons $\mathrm{X}$ et, pour quelques applications, des électrons ou des particules bêta. Les rayonnements de type proton ou neutron, qui ne concernent que de rares sites, ne seront pas considérés dans cet exposé.

Les dosimètres individuels classiques, utilisés pour la radioprotection en milieu hospitalier, peuvent se classer en 2 catégories : les dosimètres « passifs » ou à lecture différée et les dosimètres « actifs " ou à lecture directe.

\subsubsection{Dosimètres passifs}

Ces dosimètres subissent lors de l'interaction avec les rayonnements une modification durable qui ne sera mesurée que dans un deuxième temps :

- Film dosimètre : c'est le dosimètre légal en France pour les travailleurs de catégorie A. Il est constitué d'une émulsion photographique composée de cristaux de bromure d'argent. Les rayonnements ionisants vont entraîner un noircissement du film proportionnel à la dose reçue. En le plaçant derrière une série d'écrans (2 pour le film réglementaire : cuivre et plomb), on obtient plusieurs plages de noircissement, dont la différence de densité optique renseigne sur la qualité du rayonnement et permet d'en tenir compte lors de l'estimation de l'équivalent de dose.

Les principales caractéristiques ainsi que les avantages et inconvénients les plus marquants des dosimètres présentés dans ce paragraphe sont regroupés dans les tableaux I et II.

- Dosimètre thermoluminescent : il est constitué d'un matériau (généralement du fluorure de lithium, $\mathrm{LiF}$ ) sous forme de poudre, de pastille ou de languette, dans lequel des charges + et - se déplacent sous l'effet des rayonnements ionisants. Le chauffage de ce matériau entraîne une émission de lumière proportionnelle à la dose reçue. Sa disponibilité sous des faibles volumes le rend particulièrement attractif pour la mesure de la dose aux extrémités ou en des points particuliers (front, cou, ...). Il peut être stérilisé sous certaines conditions.

(1) OPRI : Office de Protection contre les Rayonnements Ionisants.

(2) ICRP : International Commission on Radiological Protection. 


\section{TABLEAU I}

Dosimètres passifs, à lecture différée, utilisés pour la surveillance individuelle. Characteristics of passive dosimeters (indirect reading) for individual survey.

\begin{tabular}{|c|c|c|}
\hline $\begin{array}{l}\text { Rayonnement } \\
\text { détecté }\end{array}$ & $\begin{array}{c}\text { rayons } \mathrm{X} \gamma \text { et } \beta \\
(E>1 \mathrm{MeV})\end{array}$ & $\begin{array}{c}\text { rayons } X \text { et } \gamma \\
\beta\end{array}$ \\
\hline Gamme de dose & de $200 \mu \mathrm{Sv}$ à $8 \mathrm{~Sv}$ & de $10 \mu \mathrm{Sv}$ à $100 \mathrm{~Sv}$ \\
\hline $\begin{array}{l}\text { Gamme d'énergi } \\
\text { (précision) }\end{array}$ & $\begin{array}{c}20 \mathrm{keV}-1 \mathrm{MeV} \\
( \pm 20 \% \text { avec filtres } \\
\text { appropriés })\end{array}$ & $\begin{array}{c}20 \mathrm{keV}-10 \mathrm{MeV} \\
( \pm \mathrm{qq} \%)\end{array}$ \\
\hline Avantages & $\begin{array}{l}\text { - identification du rayonnement } \\
\text { par filtres } \\
\text { - information conservée } \\
\text { - bon marché } \\
\text { - petite taille } \\
\text { - maniable, peu fragile } \\
\text { - information sur l'homogénéité } \\
\text { du champ de rayonnement }\end{array}$ & $\begin{array}{l}\text { - bonne réponse en fonction } \\
\text { de l'énergie } \\
\text { - sensibilité élevée } \\
\text { - « équivalent tissu } \\
\text { - petite taille (dose extrémités) } \\
\text { - réutilisable }\end{array}$ \\
\hline Inconvénients & $\begin{array}{l}\text { - sensible à la chaleur et } \\
\text { aux agents chimiques } \\
\text { - non réutilisable } \\
\text { - insensible } \beta \\
(E>1 \mathrm{MeV})\end{array}$ & $\begin{array}{l}\text { - sensible a la chaleur } \\
\text { et aux agents chimiques } \\
\text { - information effacée après } \\
\text { lecture }\end{array}$ \\
\hline
\end{tabular}

\subsubsection{Dosimètres actifs}

Le détecteur de ces dosimètres fournit, sous l'effet des rayonnements ionisants, un signal électrique permettant la mesure et l'affichage instantanés de la dose équivalente et/ou de son débit. Ces dosimètres diffèrent selon le type de détecteur : chambre d'ionisation, compteur GM ou semi-conducteur, et selon l'électronique qui leur est associée.

- Stylo dosimètre : c'est le plus simple et le plus ancien des dosimètres actifs. Le modèle le plus répandu est constitué d'une chambre d'ionisation reliée à un condensateur. La décharge de ce condensateur sous l'effet du rayonnement entraîne le déplacement du fil d'un électromètre. La position du fil sur une échelle renseigne sur la dose reçue.

- Dosimètre électronique : bien que le stylo dosimètre puisse se classer dans cette catégorie, nous réserverons cette appellation aux dosimètres équipés d'un GM ou d'un semi-conducteur et associés à une électronique pouvant contenir un micro-processeur pour les plus récents. 


\section{TABLEAU II}

Dosimètres actifs, à lecture directe, utilisés pour la surveillance individuelle. Characteristics of active dosimeters (direct reading) for individual survey.

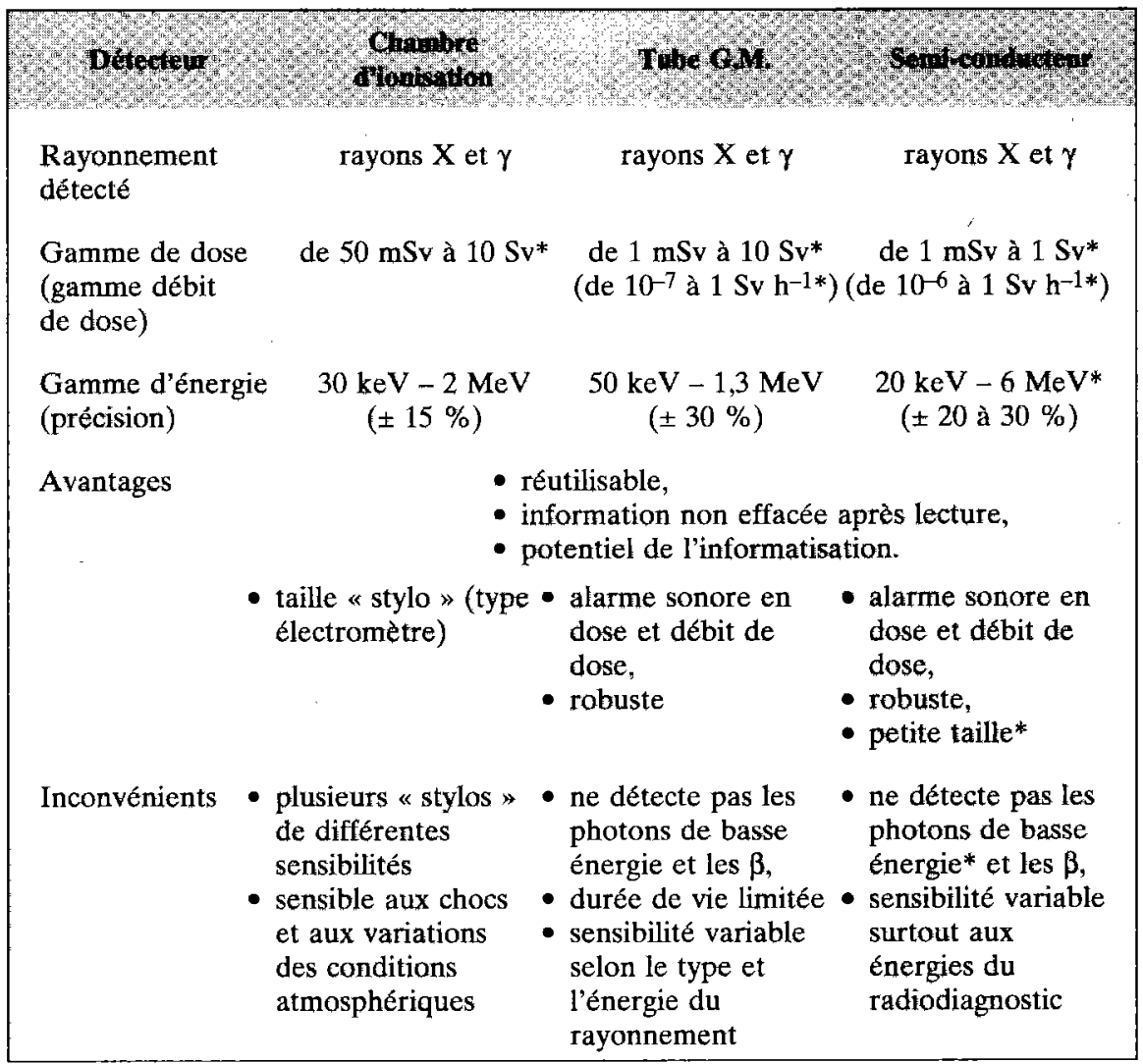

* Fonction du modèle

Ces dosimètres possèdent de nombreuses fonctions complémentaires telles que des alarmes pré-réglables sonores et visuelles, en dose ou en débit. De plus, le stockage des données sur une grande période permet la connaissance précise de la dose journalière et mensuelle voire même par tranche horaire dans une journée. Ces dosimètres constituent un outil de choix pour une dosimétrie opérationnelle efficace et performante. 


\section{Origine de l'exposition}

\subsection{Caractéristiques des sources de rayonnement}

La radiothérapie externe recouvre l'utilisation de sources telles que celles de la contacthérapie (appareils produisant des rayons $\mathrm{X}$ de faible énergie, inférieure à $50 \mathrm{keV}$ ), de la cobalthérapie (sources de cobalt 60 de plusieurs térabecquerels) et de la thérapie par photons et électrons de haute énergie (jusqu'à $25 \mathrm{MV}$ ). Sauf en contacthérapie, les niveaux d'irradiation dans la salle pendant le traitement ne permettent pas au personnel d'y rester.

En curiethérapie les principales sources utilisées sont le ${ }^{192} \mathrm{Ir}$ et le ${ }^{137} \mathrm{Cs}$. Ces sources sont appliquées dans ou au contact d'une tumeur. Le temps de l'application est de quelques jours pendant lesquels le patient est hospitalisé dans une chambre protégée. Ces sources ont des activités de l'ordre de quelques centaines à quelques milliers de mégabecquerels et produisent des débits de dose de l'ordre de quelques centaines de $\mu \mathrm{Sv} \mathrm{h}^{-1}$ à un mètre. Les sources de ${ }^{137} \mathrm{Cs}$ sont largement utilisées en curiethérapie gynécologique. Elles sont automatiquement isolées dans un stockeur par un système mécanique pour permettre l'accès des personnels soignants auprès du patient. A l'inverse, les sources de 192Ir sont implantées dans les techniques dites de « bas débit » pendant toute la durée de l'application. Les particularités de cette dernière technique résident dans la proximité de l'opérateur vis-à-vis des sources, soit lors de leur préparation soit lors de leur mise en place sur le malade ou pendant les soins prodigués lors de l'hospitalisation. Il est à noter que ces sources ne peuvent pas faire l'objet d'une protection de principe comme en radiothérapie externe.

En radiodiagnostic et en radiologie interventionnelle, les faisceaux de rayons $\mathrm{X}$ sont le plus généralement produits à une tension comprise entre 25 et $150 \mathrm{kV}$. Dans la majorité des cas, l'opérateur peut rester dans la salle où est installé l'appareil, derrière un paravent de protection. Cependant, plusieurs examens très spécialisées peuvent imposer la présence d'un opérateur près du patient pendant la radioscopie ou la prise de clichés. La protection est assurée par l'appareil lui-même ; en effet l'irradiation ne se produit que dans la direction souhaitée grâce à la gaine et à la collimation (niveau de fuites faisant l'objet de normes). Signalons également que si la plupart des techniques diagnostiques sont réalisées dans des locaux spécifiques, il existe des générateurs mobiles dans les blocs opératoires, pour les radiographies au lit, pour les urgences, ... Pour l'opérateur, le risque le plus important provient du rayonnement diffusé par le patient et éventuellement par des accessoires ou des parois. Dans les situations où le médecin est tout proche du patient, comme cela est le cas en radiologie interventionnelle, il est possible de protéger l'organisme à l'aide de tabliers plombés et les yeux par des lunettes de protection. Cependant les mains peuvent se trouver, dans certaines situations, directement dans le faisceau primaire.

En médecine nucléaire les sources sont préparées et manipulées manuellement et, après injection, le patient est porteur de la source. A l'heure actuelle, 
la grande majorité des examens à but diagnostique sont à base de $99 \mathrm{~m} \mathrm{Tc}$, avec des activités comprises entre $40 \mathrm{MBq}$ et $1 \mathrm{GBq}$. Signalons aussi l'emploi de radionucléides tels que le ${ }^{51} \mathrm{Cr}$, le ${ }^{59} \mathrm{Fe}$, le ${ }^{67} \mathrm{Ga}$, le ${ }^{123} \mathrm{I}$, le ${ }^{131} \mathrm{I}, \ldots$. On retrouve le 131I en utilisation thérapeutique, pour laquelle on peut employer jusqu'à $4 \mathrm{GBq}$ et plus. Dans les laboratoires où sont pratiquées des études in vitro, le risque lié à l'exposition externe se situe à un niveau beaucoup plus faible en raison de la manipulation de radionucléides émetteurs bêta $\left({ }^{3} \mathrm{H},{ }^{14} \mathrm{C},{ }^{35} \mathrm{~S}\right)$ ou gamma de faible énergie (125I) et d'activité peu élevée. Le ${ }^{32} \mathrm{P}$, également utilisé, émet un rayonnement bêta très énergétique $(1,7 \mathrm{MeV})$ vis-à-vis duquel on peut facilement se protéger.

\subsection{Parc radiologique médical}

Avant d'analyser les données concernant l'exposition professionnelle, il nous parait intéressant d'examiner la répartition des sources d'irradiation selon leurs applications et leur évolution de 1988 à 1995. Ces données sont présentées dans le tableau III.

TABLEAU III

Parc radiologique médical français (secteur public et assimilé et secteur libéral regroupés) et son évolution de 1988 à 1995 , d'après les statistiques officielles de l'OPRI (ACRIM, 1994).

Radiological medical park in France (public and private field combined) and its evolution from 1988 to 1995 , based on official OPRI data (ACRIM, 1994).

\begin{tabular}{|c|c|c|c|c|}
\hline 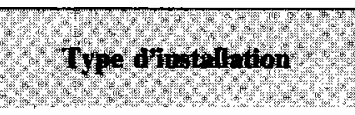 & 1908 & 1092 & Host & pord \\
\hline Radiodiagnostic & & & & \\
\hline Radioseopie & 2509 & 2027 & 1224 & 0,49 \\
\hline Radiodiagnostic léger & 3723 & 3636 & 3873 & 1,04 \\
\hline Radiodiagnostic lourd & 12882 & 13045 & 12502 & 0,97 \\
\hline Mammographie & 984 & 1913 & 2531 & 2,57 \\
\hline Scanographie & 229 & 403 & 543 & 2,37 \\
\hline Dentaire & 31249 & 34969 & 36545 & 1,17 \\
\hline Radiothérapie & & & & \\
\hline Accélérateurs & 118 & 178 & 237 & 2,01 \\
\hline Cobaltothérapie & 210 & 175 & 124 & 0,59 \\
\hline Curiethérapie & 116 & 116 & 118 & 1,02 \\
\hline Médecine nucléaire & & & & \\
\hline In vivo & 14 & 59 & 81 & 5,79 \\
\hline In vitro & 86 & 80 & 81 & 0,94 \\
\hline In vivo \& in vitro & 104 & 104 & 105 & 1,01 \\
\hline
\end{tabular}


On peut relever que sur cette période de 7 ans :

- près de $64 \%$ du parc d'appareils de radiodiagnostic est constitué par des installations dentaires et tous les appareils hors radiodiagnostic ne représentent que $1 \%$ de l'ensemble du parc ;

- la radioscopie est en diminution (-51\%) suite à la suppression des actes de radioscopie thoracique de la nomenclature et au non-renouvellement des autorisations (Directive européenne sur la protection du patient, 1984) ;

- la mammographie progresse de façon spectaculaire $(+157 \%$ ) de même que la scanographie $(+\mathbf{1 3 7} \%)$ alors que les autres techniques de radiodiagnostic médical restent stables et que le radiodiagnostic dentaire progresse régulièrement $(+17 \%)$;

- en radiothérapie les accélérateurs progressent et sont plus nombreux, depuis 1992, que les appareils de cobalt dont le nombre ne cesse de décroître ;

- la curiethérapie reste stable en nombre de projecteurs de source. Notons cependant l'apparition récente de la curiethérapie haut débit dont le nombre d'installations est à ce jour limité ;

- enfin, la médecine nucléaire progresse aussi bien dans le domaine de l'in vitro que de l'in vivo.

\section{Surveillance individuelle}

\subsection{Bilan de la surveillance réglementaire}

Un panorama national de l'exposition professionnelle en 1995, selon les principales utilisations médicales des rayonnements ionisants et pour les travailleurs du milieu médical surveillés par l'OPRI, est présenté dans le tableau IV. Le nombre de travailleurs surveillés et exposés (ceux dont le film a été exposé au moins une fois dans l'année au-dessus du niveau d'enregistrement de 0,2 mSv) ainsi que la dose annuelle moyenne au corps entier selon la population considérée, et la dose collective pour les différentes applications médicales des rayonnements ionisants y sont détaillés.

Plusieurs éléments peuvent être relevés à partir de ce tableau :

- $4,5 \%$ des travailleurs surveillés peuvent être considérés comme significativement exposés ( $H>0,2 \mathrm{mSv}$ au moins pour un mois);

- $84,4 \%$ des personnes surveillées travaillent dans le domaine du radiodiagnostic, $8,6 \%$ dans celui de la radiothérapie et 9,0\% dans celui des sources non scellées (médecine nucléaire);

- les travailleurs exposés $(H>0,2 \mathrm{mSv})$ se répartissent en 68,17 et $15 \%$, respectivement pour ces 3 domaines ;

- la dose collective annuelle se répartit entre ces 3 domaines respectivement pour 74,16 et $10 \%$; 
- le radiodiagnostic dentaire représente $22,5 \%$ des travailleurs surveillés, mais seulement $12,9 \%$ des travailleurs exposés et $8,4 \%$ de la dose collective.

Il est intéressant d'examiner également la répartition des doses corps entier. Les données concernant l'année 1995 sont présentées dans le tableau V selon la gamme de dose et l'application médicale. On remarque que $93 \%$ et $99 \%$ des travailleurs surveillés sont exposés, respectivement, à des niveaux inférieurs au niveau d'enregistrement $(0,2 \mathrm{mSv})$ et à $5 \mathrm{mSv}$ (limite annuelle actuelle pour le public); et que parmi les travailleurs exposés ( $>0,2 \mathrm{mSv})$ moins de $0,4 \%$ reçoivent une dose équivalente annuelle supérieure à la limite actuelle de $50 \mathrm{mSv}$.

L'ensemble de ces données montre clairement que, globalement, au moins $99 \%$ des travailleurs de catégorie $\mathrm{A}$ sont soumis à des expositions annuelles dont le niveau est inférieur à $15 \mathrm{mSv}$, valeur au-dessous de laquelle une surveillance individuelle systématique n'est pas nécessaire (catégorie B). Quant à la limite des $50 \mathrm{mSv}$ elle n'est dépassée que dans moins de $0,1 \%$ des cas.

Les données des tableaux IV et $\mathrm{V}$, dans la mesure où elles sont représentatives de l'exposition des travailleurs (film dosimètre effectivement porté et cor-

TABLEAU IV

Bilan de l'exposition professionnelle des travailleurs du milieu médical en 1995 surveillés par l'OPRI (source OPRI).

Occupational exposure of workers in medical field in 1995 supervised by OPRI.

\begin{tabular}{|c|c|c|c|c|c|}
\hline \multirow{2}{*}{$\begin{array}{l}\text { Applicothons } \\
\text { medicales } \\
\text { m }\end{array}$} & \multicolumn{2}{|c|}{$\begin{array}{l}\text { Nombre } \\
\text { de travailleurs }\end{array}$} & \multicolumn{2}{|c|}{$\begin{array}{l}\text { Dose conp entier } \\
\text { moyenne anauelle (nSv) } \\
\text { par travalleur }\end{array}$} & \multirow{2}{*}{$\begin{array}{l}\text { Dose } \\
\text { collective } \\
\text { onmuelte } \\
\text { (hSV) }\end{array}$} \\
\hline & simelles & exposés" & swredle & exposet & \\
\hline \multicolumn{6}{|l|}{ Radiodiagnostic } \\
\hline Hôpital & 38311 & 1182 & 0,08 & 2,70 & $\mathbf{3 , 1 9}$ \\
\hline Privée ${ }^{* *}$ & 14682 & 1027 & 0,17 & 2,41 & 2,48 \\
\hline Dentaire & 16949 & 436 & 0,05 & 2,11 & 0,92 \\
\hline Médecine du travail & 4825 & 87 & 0,04 & 2,07 & 0,18 \\
\hline $\begin{array}{l}\text { Radiothérapie } \\
\text { (externe et curiethérapie) }\end{array}$ & 7623 & 670 & 0,19 & 2,21 & 1,48 \\
\hline \multicolumn{6}{|l|}{ Médecine nucléaire } \\
\hline In vivo & 3653 & 518 & 0,24 & 1,66 & 0,86 \\
\hline In vitro & 4669 & 102 & 0,02 & 0,90 & 0,09 \\
\hline Total & 90712 & 4022 & 0,10 & 2,29 & 9,20 \\
\hline
\end{tabular}

* Dose mensuelle supérieure à $0,2 \mathrm{mSv}$

** Médecine générale privée + médecine spécialisée et clinique + cabinets de radiologie privée 
TABLEAU V

Répartition des équivalents de dose annuels au corps entier pour l'année 1995 (source OPRI).

Annual dose equivalent distribution to the whole body for 1995.

\begin{tabular}{|c|c|c|c|c|c|c|c|}
\hline \multicolumn{8}{|l|}{ 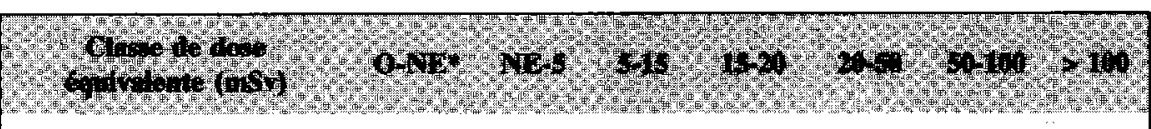 } \\
\hline \multicolumn{8}{|l|}{ Radiodiagnostic } \\
\hline Hôpital & 37129 & 1048 & 100 & 5 & 22 & 6 & 1 \\
\hline Privé & 13655 & 924 & 79 & 5 & 17 & 3 & 0 \\
\hline Dentaire & 16513 & 405 & 24 & 2 & 3 & 2 & 0 \\
\hline Médecine du travail & 4738 & 78 & 8 & 1 & 0 & 0 & 0 \\
\hline $\begin{array}{l}\text { Radiothérapie } \\
\text { (externe et curiethérapie) }\end{array}$ & 6953 & 601 & 58 & 3 & 9 & 0 & 0 \\
\hline \multicolumn{8}{|l|}{ Médecine nucléaire } \\
\hline In vivo & 3135 & 487 & 28 & 1 & 2 & 0 & 0 \\
\hline In vitro & 4567 & 101 & 1 & 0 & 0 & 0 & 0 \\
\hline Total & 86690 & 3644 & 298 & 17 & 53 & 11 & 1 \\
\hline
\end{tabular}

* Niveau d'enregistrement (NE, 0,2 mSv)

rectement positionné, ...), rassurent quant au niveau d'exposition externe, et donc de protection, du personnel dans le milieu médical au plan national. Néanmoins certains travailleurs, soit en raison de leurs conditions de travail, soit par le fait que la dosimétrie réglementaire ne rend pas exactement compte de leur exposition réelle, doivent pouvoir bénéficier d'une surveillance complémentaire dans le cadre de la dosimétrie opérationnelle. Ce sont des données relatives à ce type de surveillance que nous rapportons ci-dessous.

\subsection{Dosimétrie opérationnelle}

La nécessité d'une dosimétrie opérationnelle est confirmée par plusieurs auteurs, spécialement quand une exposition très élevée des mains peut survenir, ce qui peut se produire lors de certains examens de radioscopie ou encore lors de la manipulation de sources radioactives en médecine nucléaire et curiethérapie. Dans ces situations le film « poignet » ne reflète qu'imparfaitement le niveau réel d'exposition.

Les résultats qui suivent se rapportent à des études menées avec des dosimètres thermoluminescents et avec des dosimètres électroniques dans les trois domaines d'applications déjà cités. 


\subsubsection{Etudes à l'aide de dosimètres TLD}

Les dosimètres utilisés se présentent soit sous la forme d'une pastille placée sur une bague, soit sous la forme d'une languette souple de petite dimension placée au bout des doigts.

\section{a) Cas de la médecine nucléaire}

Etude de Villejuif (Aubert et al., 1991) : les dosimètres ont été portés au doigt le plus exposé pendant 15 jours par les techniciens préparant les solutions radioactives (en moyenne $20 \mathrm{GBq}$ - $600 \mathrm{mCi}$ - de $99 \mathrm{mTc}$ par jour) pour les explorations diagnostiques en médecine nucléaire. Sur la période de 2 semaines, un équivalent de dose de $9,4 \mathrm{mSv}$ a été mesuré. En extrapolant à l'année (46 semaines de travail), on obtient un équivalent de dose d'environ $220 \mathrm{mSv}$, soit près de la moitié de la limite annuelle réglementaire pour les extrémités, si cette activité n'était pratiquée que par une seule personne, ce qui en pratique n'est pas le cas.

Résultats de la littérature (Batchelor et al., 1991 ; Schürnbrand et al., 1982) : de nombreuse études ont attiré l'attention sur les risques d'exposition élevés au niveau des mains lors de la préparation et de l'injection des radiopharmaceutiques. Pour le $99 \mathrm{~m} T \mathrm{c}$, la valeur maximale au niveau des doigts est de l'ordre de $3 \mu \mathrm{Sv} \min ^{-1} \mathrm{MBq}^{-1}$. En fonction de l'activité du service, cette valeur peut conduire à une exposition annuelle proche, et parfois même supérieure à la limite de $500 \mathrm{mSv}$ pour les extrémités. L'utilisation de protège-seringues s'impose donc, surtout depuis l'apparition de tels dispositifs en tungstène, ce qui les rend plus pratiques d'emploi tout en offrant une réduction du débit d'un facteur d'environ 60 .

\section{b) Cas de la radiologie interventionnelle}

Etude de Villejuif (Aubert et al., 1991 ; Sapoval et al., 1992) : un dosimètre TLD a été placé stérilement sur les annulaires gauche et droit de l'opérateur, sur le front et sur la thyroïde non protégés. Les examens réalisés ont consisté en des procédures dites éloignées (PE) où l'opérateur et ses mains sont généralement à distance du faisceau incident (artériographie, embolisation, ...) ainsi qu'en des procédures dites rapprochées (PR) où les mains sont proches du faisceau, voire parfois dans le faisceau direct (drainage biliaire, pyélostomie, ...). Les dosimètres étaient changés à chaque procédure et les temps de scopie étaient mesurés (scopie totale et scopie avec une partie de la main dans le faisceau direct). Les résultats concernant l'exposition des organes étudiés, ainsi que le nombre d'examens maximal autorisés par an compte tenu des limites annuelles d'exposition réglementaires, figurent dans le tableau VI.

Etude d'Orléans (Germanaud et al., 1993) : dans le cadre de cholangio-pancréatographies rétrogrades endoscopiques (CPRE), l'exposition de l'opérateur en différents points de l'organisme a été évaluée à l'aide de dosimètres TLD portés pendant un mois au cours duquel 24 interventions ont été pratiquées, correspondant à un temps total de scopie de 164 min et à la réalisation de 170 clichés. Les principaux résultats de cette étude sont rapportés dans le tableau VII. 
TABLEAU VI

Equivalent de dose moyen aux organes étudiés lors d'un examen rapproché $(P R)$ et d'un examen éloigné $(P E)$, ainsi que le nombre maximum d'examens autorisés au regard des limites d'exposition en vigueur.

Mean dose equivalent to different organs during close (PR)

and remote (PE) examination and maximal number of examination authorised by the present legislation.

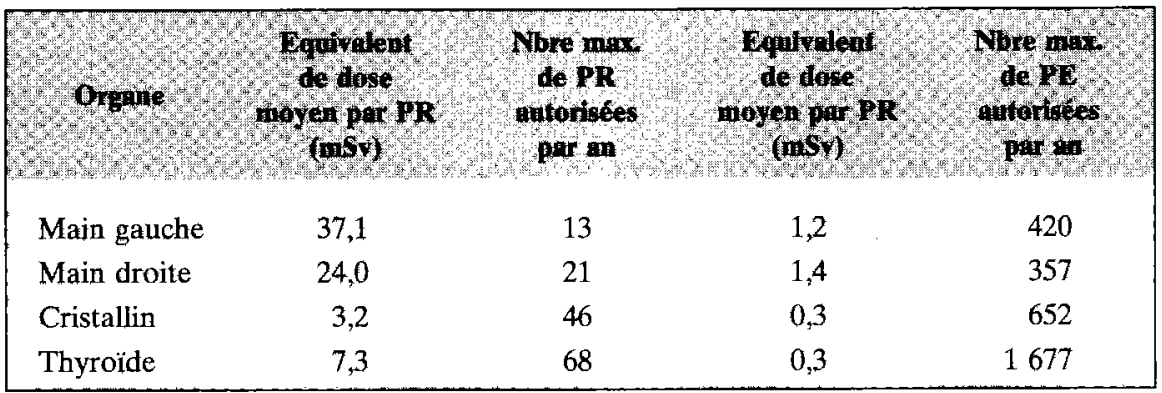

TABLEAU VII

Équivalent de dose mesuré par TLD en différentes localisations pour un opérateur pratiquant des CPRE.

Dose equivalent measured with thermoluminescent dosimeter at various locations for an operator during RECP.

\begin{tabular}{|c|c|}
\hline Hoolistion do TTP & Aprivalent do dose (mS p par pols) \\
\hline Front (dose cristallin) & 3,2 \\
\hline Cou (dose thyroïde) & 2,4 \\
\hline Doigt (main gauche) & 0,9 \\
\hline Sur le tablier (épaule) & 2,4 \\
\hline Sous le tablier & $<0,1$ \\
\hline
\end{tabular}

Résultats de la littérature (Amiel et al., 1977; Cruikshang et al., 1980; Gustafsson et Lunderquist, 1981 ; Jeans et al., 1985 ; Johnson et al., 1992 ; Santen et al., 1981) : dans le domaine de la radiologie interventionnelle, de nombreuses études ont été publiées. Les résultats d'une partie d'entre elles ont été regroupés dans le tableau VIII. Ils concernent l'exposition du cristallin, de la thyroïde et des mains dans le cas de procédures proches ou rapprochées.

\subsubsection{Etude à l'aide de dosimètres électroniques (Aubert et al., 1994)}

Les dosimètres utilisés, qui contiennent un petit détecteur (diode silicium compensée en énergie) associé à une électronique intégrée, se présentent sous un format réduit (dimension d'une carte de crédit). Ils bénéficient de toutes les 
TABLEAU VIII

Données de la littérature concernant l'exposition de certaines parties du corps liée à des pratiques de radiologie interventionnelle.

Literature data concerning exposure of body parts in interventional radiology.

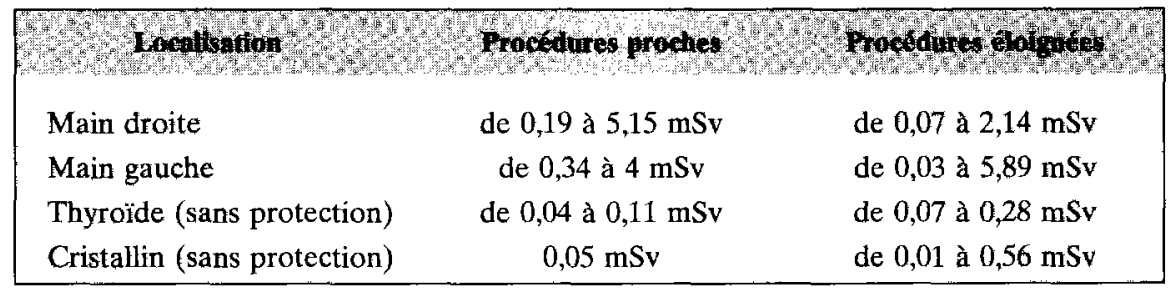

fonctionnalités attendues d'un tel appareil pour l'application envisagée (lecture directe, dose, débit de dose, alarmes sonore et visuelle). De plus l'électronique intégrée permet la gestion des informations dosimétriques (intégration de la dose par mois, par an, ...) et les échanges avec un système d'exploitation externe (Lacoste et Lucas, 1993).

Le tableau IX présente pour un certain nombre de postes la valeur moyenne de l'équivalent de dose moyen par jour d'activité, la gamme de ces valeurs sur la période d'observation (de 3 à 9 semaines) et l'équivalent de dose moyen annuel extrapolé sur la base de 220 jours de travail par an. Ces dosi-

\section{TABLEAU IX}

Equivalent de dose au personnel travaillant aux postes les plus exposés. Dose equivalent to personnel working at the most exposed locations.

\begin{tabular}{|c|c|c|c|}
\hline 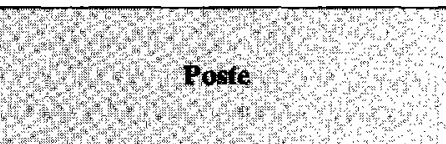 & 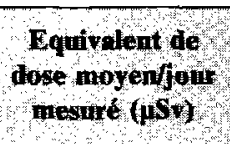 & $\begin{array}{l}\text { Gamme } \\
\text { deralew } \\
\text { (pory) }\end{array}$ & $\begin{array}{l}\text { Eopryalert do } \\
\text { dose nogyengo } \\
\text { estine (nsh) }\end{array}$ \\
\hline Injection scinti. osseuse & 38,3 & $12,8-109$ & 8,4 \\
\hline Injection scinti. thyroìdienne & 3,1 & $\approx 0-14,3$ & 0,7 \\
\hline Manipulateur médecine nucléaire & 3,4 & $0,9-8,1$ & 0,8 \\
\hline Technicien laboratoire chaud & 6,2 & $\approx 0-61,0$ & 1,4 \\
\hline Manipulateur curiethérapie & 12,3 & $\approx 0-133$ & 2,7 \\
\hline $\begin{array}{l}\text { Opérateur radiologie interv. } \\
\text { (sous tablier) }\end{array}$ & 20,5 & $\approx 0-218$ & 4,5 \\
\hline $\begin{array}{l}\text { Opérateur radiologie interv. } \\
\text { (à l'épaule) }\end{array}$ & 186,1 & $\approx 0-1759$ & 41,0 \\
\hline
\end{tabular}


mètres permettent également de connaître la valeur des dépassements de seuils, en dose ou débit de dose, préalablement choisis, ainsi que la durée de ces dépassements.

\section{Conclusion}

Dans le cadre des risques d'exposition liés aux différentes activités pratiquées en milieu hospitalier et dans les laboratoires, la surveillance opérationnelle est un complément indispensable à la surveillance réglementaire telle qu'elle est habituellement pratiquée. En effet, si cette dernière couvre la grande majorité des besoins dans les situations de risque d'exposition externe de l'organisme, certaines applications particulières nécessitent la mise en cuvre d'une surveillance complémentaire qui doit essentiellement porter sur l'utilisation de dosimètres additionnels. L'évaluation directe au niveau de l'opérateur, des expositions reçues en fonction de la nature du geste médical, permet de mieux le sensibiliser vis-à-vis du niveau de risque potentiel et de le motiver pour utiliser des protections particulières (protège-thyroïde, lunettes, ...). Elle permet aussi d'estimer un nombre maximal d'interventions possibles par an et par personne.

Les études menées par différentes équipes ont montré que d'une façon générale les personnes les plus exposées sont :

- les manipulateurs en curiethérapie par les sources d'iridium 192 ;

- les préparateurs de sources non scellées en médecine nucléaire ;

- les médecins des services d'hémodynamique;

- les médecins pratiquant la radiologie vasculaire ;

- les médecins gastro-entérologues pratiquant le cathétérisme des voies biliaires.

A l'heure actuelle il est certain que la radiologie interventionnelle constitue la pratique où le risque d'exposition du personnel est le plus élevé, pouvant même conduire à approcher ou dépasser les limites réglementaires.

Ces constatations doivent nous inciter à réfléchir sur plusieurs aspects de la pratique de la radioprotection en milieu hospitalier :

- la multiplicité des postes concernés par une surveillance particulière doit conduire la personne compétente en radioprotection à s'appuyer sur des relais dans les différents services concernés (personnel d'encadrement, manipulateur, ...);

- la nécessité d'une collaboration de qualité entre le médecin du travail et la personne compétente ;

- le respect des limites d'exposition, surtout dans la perspective de la mise en place des recommandations plus contraignantes de la CIPR 60 (1977);

- l'application du principe d'optimisation dans le domaine médical. 


\section{RÉFERENCES}

Amiel M., Sobotka F., Philippon L. (1977) Irradiation du médecin, du patient et du personnel médical dans les explorations cardiaques et vasculaires. J. Radiol. Electrol. Med. Nuc. 58, 815-816.

Annuaire de la Cancérologie/Radiothérapie et des Imageries Médicales en France (1994) (ACRIM), 14e édition, éditeur A. Laugier, Paris.

Aubert B., Lamon A., Beaudet G. (1994) Evaluation de nouveaux dosimètres électroniques en milieu hospitalier. Journées SFRP de La Rochelle, 20-21 septembre 1994.

Aubert B., Nguyen J., Sapoval M. (1991) Evaluation des expositions en médecine nucléaire et radiologie interventionnelle. In : Recueil des communications du $X X X^{e}$ congrès de la SFPH. Rennes, 6-8 juin 1991, pp. 213-210.

Batchelor S., Penfold A., Aric I., Huggins R. (1991) Radiation dose to the hands in nuclear medicine. Nucl. Med. Commun. 12, 439-444.

Cruikshang J.G., Fraser G.M., Laws J. (1980) Finger doses received by radiologist during chiba needle percutaneous cholangiography. Br. J. Radiol. 53, 584-585.

Directive européenne (1984) 84/466/Euratom du 3 septembre 1984 sur la protection radiologique du patient.

Germanaud J., Legoux J.L., Sabattier R., Causse X., Trinh DH (1993) Radioprotection des opérateurs lors des cholangio-pancréatographies rétrogrades endoscopiques. Gastroenterol. Clin. Biol. 17, 259-263.

Gustafsson M., Lunderquist A. (1981) Personnal exposure to radiation at some angiographic procedures. Radiology 140, 807-811.

ICRP (1977), Recommandations of the International Commission on Radiological Protection, ICRP Publication 26, Pergamon Press, Oxford.

ICRP (1991), Recommandations of the International Commission on Radiological Protection, ICRP Publication 60, Pergamon Press, Oxford.

ICRU (1985) Détermination des Equivalents de Dose dus aux Sources Externes de Rayonnement, rapport $n^{\circ} 39$.

ICRU (1988) Determination of Dose Equivalents of External Radiation Sources, Part 2, rapport $n^{\circ} 43$.

ICRU (1992) Measurement of Dose Equivalents of External Photon and Electron Radiations, rapport $\mathrm{n}^{\circ} 47$.

ICRU (1993) Quantities and Units in Radiation Protection Dosimetry, Rapport $\mathbf{n}^{\circ} 51$.

ISO 4037 (1992) $\mathrm{X}$ and gamma reference radiations for calibrating dosemeters and dose ratemeters and for determining their response as a function of photon energy, Part 1 et 2 .

Jeans S.P., Faulkner K., Love M.G., Bardsley RA (1985) An investigation of the radiation dose to staff during cardiac radiological studies. Br. J. Radiol. 58, 419-428.

Johnson L.W., Moore R.J., Balter S. (1992) Review of radiation safety in the cardiac catheterization laboratory. Catheterization and Cardiovascular Diagnosis 25, 186-194.

Lacoste F., Lucas M. (1993) Le système Dosicard. Radioprotection 28, 77-81.

Santen B.C., Kan K., Velthuyse H.J.M., Julius H.W. (1981) Exposure of the radiologist to scattered radiation during angiography. Radiology 140, 807-811.

Sapoval M., Aubert B., Nguyen J., Ricard M., Roche A. (1992) Radioprotection in interventional radiology : should we be concerned? Radiology 185P, 364.

Schürnbrand P., Schicha H., Thal H., Emrich D. (1982) External radiation exposure of personnel working with ${ }^{99} \mathrm{~m}$ Technetium. Eur. J. Nucl. Med. 7, 237-239. 